\section{MiSPIA: Microelectronic Single-Photon 3D Imaging Arrays for low-light high-speed Safety and Security Applications}

\section{D cameras, based on the measurement of the Time-of-Flight of single photons by means of Single-Photon Avalanche Diode imagers, for automotive safety driving and security surveillance application}

MiSPIA will develop beyond state-of-the-art photonics technology for array imagers of smart-pixels able to detect single photons. Intelligent in-pixel pre-processing will simultaneously provide ultra high sensitivity (single-photon level), very high frame-rate (up to 200,000fps) and advanced multi-spectral (300-900nm) three-dimensional (3D) distance ranging and two-dimensional (2D) imaging of fast moving objects. MiSPIA detectors will be used in two key applications: long-range (200-1,000m) 2D and 3D active identification in low light level surveillance operations; and very fast (over 200fps) short-range (10-50m) 3D monitoring in automotive pre-crash safety systems. Instead of (slow and noisy) CCDs and CMOS active pixels (with poor sensitivity and noisy electronics), MiSPIA will exploit the ultimate performances of truly-single photon detectors: the Single-Photon Avalanche Diodes (SPAD). MiSPIA imagers will be based on four different SPAD smart-pixels: "photon-counting" pixels for 2D imaging; LIDAR pixels for 3D direct "time-of-flight" (dTOF); two different phase-sensitive pixels for 3D indirect time-of-flight (iTOF) depth acquisitions. Full-size imager chips will be manufactured, characterized and eventually integrated into two 3D ranging cameras deployed into the two end-users applications for validation.MiSPIA technologies will be both highly-advanced and cost-effective: a high-voltage $0.35 \mu \mathrm{m}$ CMOS processing for front-side illuminated imagers; and a new flipped-chip Silicon-on-Insulator (SOI) CMOS technology for back-side illuminated imagers. Both will prove beyond state-of-the art co-integration of photonic SPAD detectors and CMOS microelectronics for intelligent and dense 2D imaging and 3D ranging high-performance cameras. Such cameras will provide imaging at the quantum limit and on-chip pre-processing at the most effective speed at a drastic reduction of manufacturing costs, down to $5 €$ per imager chip.The developments of the MiSPIA Project will be published on the official website www.mispia.eu.

\section{Contact info}

ZAPPA Franco

Via Golgi 40

20133 Milano

ITALY

Tel. +390223996149

Fax. +39022367604

E-mail: franco.zappa@polimi.it

\section{Participants}

Coordinator: POLITECNICO DI MILANO, ITALY

Participating partners:

\begin{tabular}{|l|l|l|}
\hline & Organisation & Country \\
\hline POLITECNICO DI MILANO & ITALY \\
\hline
\end{tabular}

\section{Project ID card}

- Funded under: Information and Communication Technologies

- Area: ICT-2009.3.7 Photonics

- Total Cost: $€ 3.40 m$

- EU Contribution: $€ 2.63 \mathrm{~m}$

- Project Reference: 257646

- Execution: From $01 / 06 / 2010$ to 31/05/2013

- Project status:

Running

- Contract type:

Information and Communication Technologies CP Collaborative Project

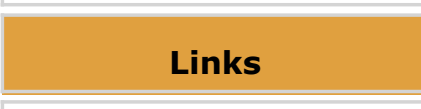

- MiSPIA

Multimedia

- Poster

JPG | 1740 kBytes

\begin{tabular}{|c|}
\hline \multicolumn{1}{|c|}{ News } \\
\hline \hline \\
\hline
\end{tabular}

Publications

- FP7-257646-MISPTA 


\begin{tabular}{|l|l|}
\hline $\begin{array}{l}\text { FRAUNHOFER-GESELLSCHAFT ZUR FOERDERUNG DER ANGEWANDTEN FORSCHUNG } \\
\text { E.V }\end{array}$ & GERMANY \\
\hline EMZA VISUAL SENSE LTD & ISRAEL \\
\hline CENTRO RICERCHE FIAT SCPA & ITALY \\
\hline CF CONSULTING FINANZIAMENTI UNIONE EUROPEA SRL & ITALY \\
\hline MICRO PHOTON DEVICES S.R.L. & ITALY \\
\hline HERIOT-WATT UNIVERSITY & KINGDOM \\
\hline
\end{tabular}

Last update: 12/12/2011 